\title{
Research on key technology of equipment maintenance material catalogue compilation management system
}

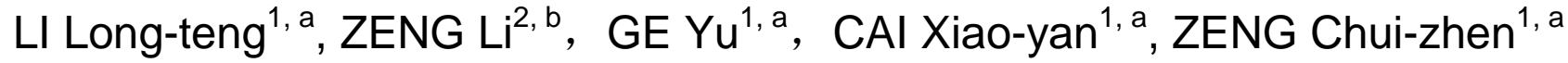 \\ ${ }^{1}$ Dept. of Command and Control, Wuhan Ordnance NCO Academy, Wuhan, China \\ ${ }^{2}$ Dept. of Language Training, Wuhan Ordnance NCO Academy, Wuhan, China \\ alltkiller@gmail.com, ${ }^{b}$ wunsche@163.com
}

Keywords: equipment maintenance material catalogue; key technology; query optimization; data report; data stream; picture storage

\begin{abstract}
To improve the low efficiency in compiling the catalogue of equipment maintenance material by manpower, the key technology in design and implementation of equipment maintenance material catalogue compilation management system are researched based on the analysis of the technology of Big Data and COM component, in which class package technology, query optimization technology, data report technology based on COM component and picture data storage technology based on data stream are contained. With .Net as a development environment, equipment maintenance material catalogue compilation management system was developed with C\# language. The information management for compiling equipment maintenance material catalogue was realized.
\end{abstract}

\section{Preface}

The catalogue of equipment maintenance material is not only used to retrieve related information in the course of regular equipment maintenance and maintenance material management, but also regarded as the fundamental basis to purchase weapon maintenance material and supply material for army.

To correspond with the demand of information and real combat construction, efforts is placed on researching on key technology of equipment maintenance material catalogue compilation management system (short for the system) to realize the information management for equipment material catalogue, which can strengthen management, decrease consumption and promote the work efficiency in and out war.

\section{System requirements analysis}

To achieve better management for the catalogue of various type of equipment maintenance material, the related parameter of equipment maintenance material in the system should be added, modified and the query census of related data should be realized. In the course of designing the system, the favorable extensibility the system must provide should be took into account due to its diverse type, huge amounts and fast update maintenance materials, requiring that a large amount of data can be retrieved and positioned quickly. Moreover, considering that great deals of maintenance material catalogue data are still stored in the system, the system should be able to import the existed data in batches. The system function module diagram is as follow (Fig.1). 


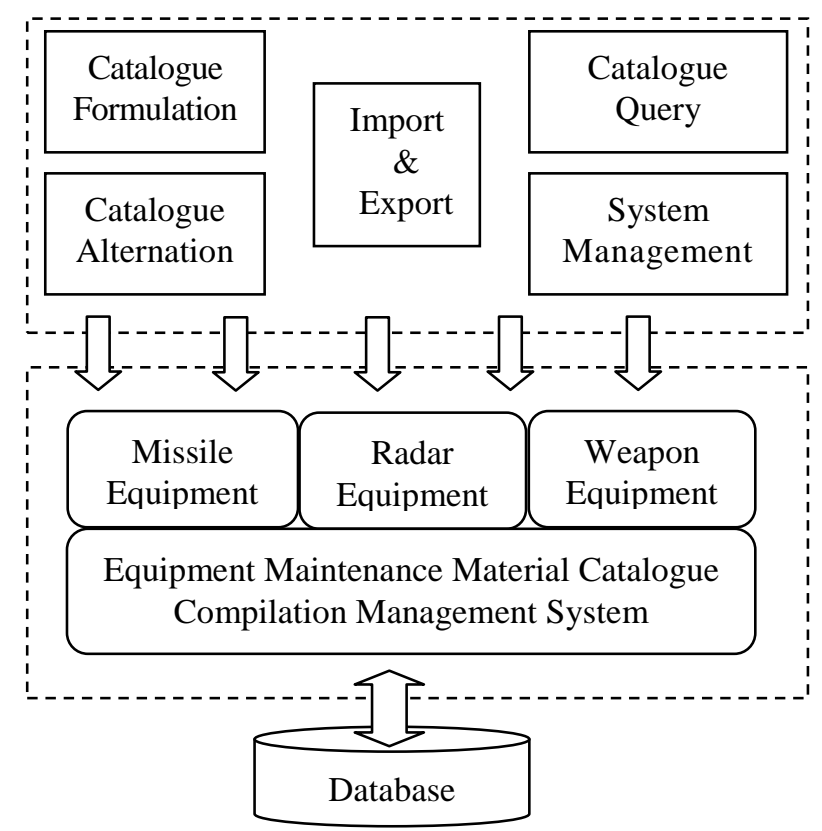

Fig.1 The system function module

\section{Key technology}

\section{A. Design the equipment class and material class}

Two classes are defined in the system: equipment class and material class.

1) Equipment class: Equipment class mainly consists of the following four events.

Check whether the same information can be looked up in database according to the input information of weapon system name, equipment name, maintenance major and user ID number. Define a return value of Boolean variable query result. If query number is greater than zero, the return value is false value. In converse, it is true value. The flow chart is shown below (Fig.2).

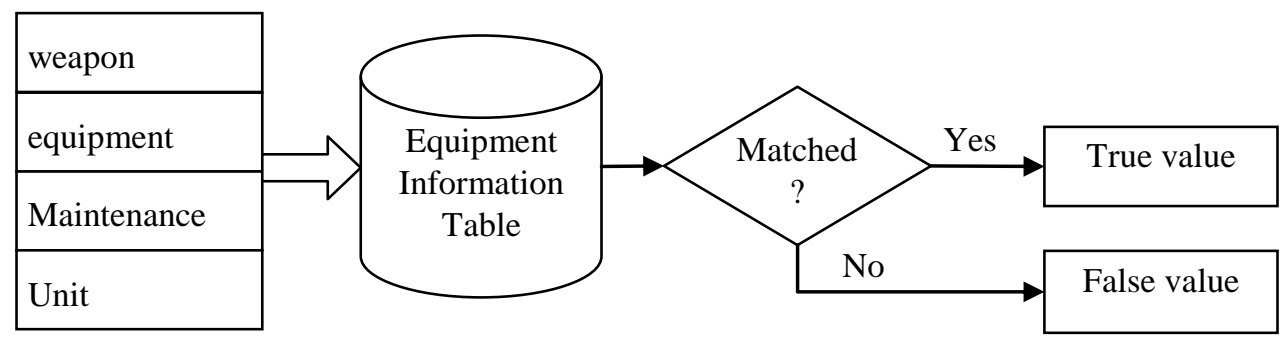

Fig.2 The flow chart of judging whether the equipment exist

In order to store and identify data conveniently in database, part information of equipment is chiefly recorded their ID number. In the practical operation course, the information class is judged by the obtained ID number. Using this method can improve the running speed of program.

Before adding new equipment information, the existence of equipment's record should be checked first. If the equipment isn't recorded, the largest equipment flow number should be found and pulsed 1, which will be used as a part of new equipment's ID number to recognize equipment's order. And then, you input the ID number and other information of the equipment in database.

If the alternation information of equipment is set in class in advance, the matched member can be selected directly in need, which can not only condense program code, but also promote program's running speed.

2) Equipment class: Equipment class mainly consists of two events: add and alter. The return values are recorded by those two Boolean variables respectively. If it can successfully add or alter, 
the return value is true. If it can't successfully add or alter, the return value is false and the system will warn the wrong reason.

\section{B. Query operation design}

Two kinds of data, equipment information and material catalogue information data, are mainly included in equipment maintenance material catalogue compilation management system. As the volume of query information is huge, the query efficiency dramatically influences the overall system performance. Therefore, how to optimize query procedure in practical query operation is a burning problem we should tackle with. Query is optimized in terms of database design and SQL language.

1) Create data storage of clustered index: Clustered index makes data in table in physical order and restored the data in disk. Using index to make query in this way is efficient. Each table can only create one clustered index. If there is no clustered index in table, SQL Server will take primary key column as clustered index key when the table creates primary key. By using clustered index, data is physically ordered in data page and the repeated data are also ordered together. Once you find the first row of key value in the limited range and its subsequent index values row can link with it physically by using language which can limit search range like (Between、 $<、<=、>、>$ ), Group by or Order by to make query, you don't need to get further search, from which the wide range search is avoided and query speed is greatly improved.

2) SQL language optimization: The first line of SQL Server is required to analyze the SQL language grammatically and transfer it into certain inner view language tree, and it is transferred into standard expression according to transformation rules. After that, using the lower lever optimizer creates specific operation calculation methods for that information whose every operation is required to select connection methods and connection order according to information's storage path, storage and analysis of data and storage data clustering in grammar tree, and then create query plans finally. There are usually more than one query operation plans. The cost of each plan is calculated, from which the minimum cost one is chosen. The procedure of SQL optimization is shown in Fig. 4.

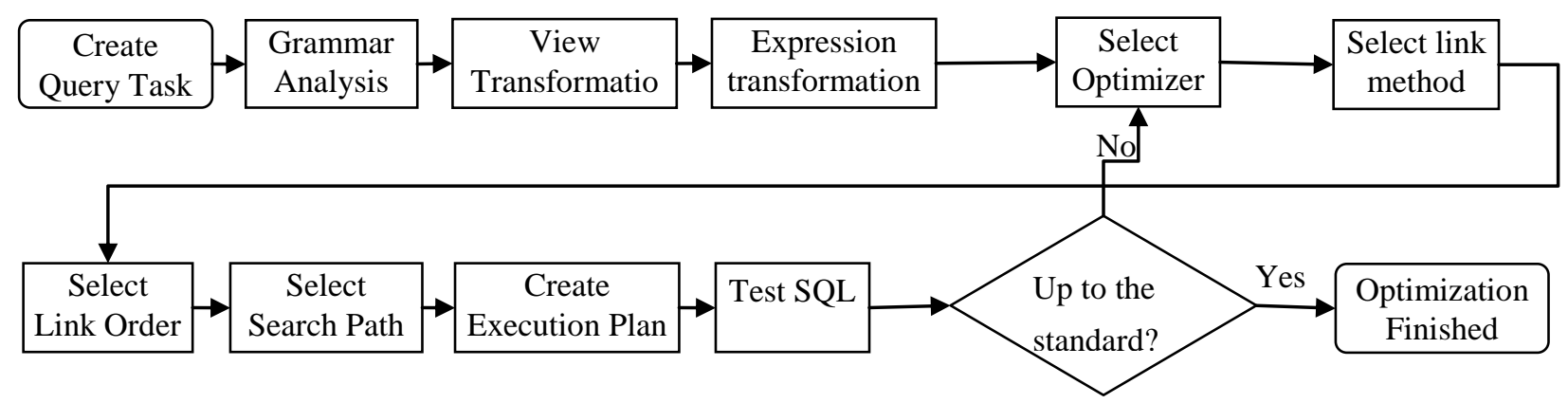

Fig.4 The flow chart of SQL optimization

\section{Data report import and export design}

Emphasis is place on whether either the recorded data or query results can be exported as Excel report in standard format, or the edited data in Excel report can be imported in database in equipment maintenance material management system, which put forward a higher requirement for the design of the system.

1) Overall structure design for system report: The design of the whole report generation system in equipment maintenance material catalogue management system abides by layered structure of software design, which is shown in Fig. 5. The function of uppermost user lever is to connect user, display reports, import data and print report. The middle layer is business logic layer, 
in which the calculation of logic, the read of report module and the filling of report data are realized. The bottom layer is regarded as data access layer and data communication layer. Data access layer connect directly with database to realize the selection, statistication and calculation of data.

As the report generation system must have high versatility, object and component technology was used to develop the system, by which COM component based on Excel was realized.

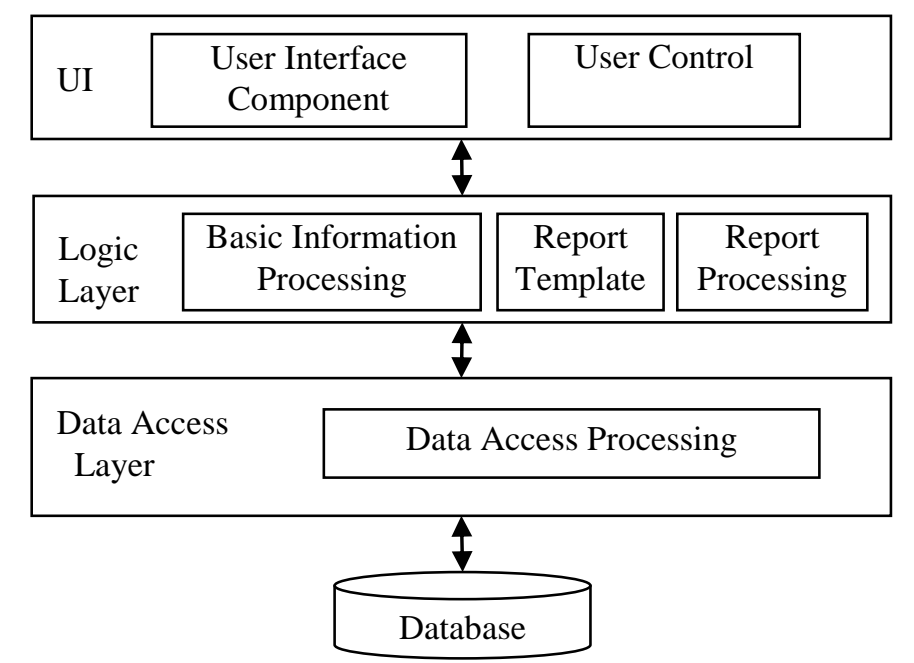

Fig.5 The system structure diagram for report generation

2) Report core class and report template: Report generates core class which is responsible for the initialization of each report. For instance, it selects the matched information of specific report from report-Manager, and creates report generation object in similar style according to report's matched information, like Card. and Style Report. After initialized, the report generation object is selected to analyze the template document and creates the related report document. Report Engine user allocates and creates binary flow by Report Engine, and then writes into Response.

Report template document determines the export format of whole report and the display method of data. The template is an Excel document in XLT format, which defined the report format of specific data generation. The header field, data field and bottom field of template should be designed. Header field can set up as fixed or dynamic format according to requirement. Report data field is an adaptive region, which can automatically expand with the increasing data. The bottom field will move downward with the change of data, which is always under the data field.

\section{Design for material picture information storage based on data flow technology}

1) Design ideas: At present, the regular way to store picture is to use link, which means that a picture storage path is established under installation path and this path is called to get the picture in the program. It accounts for a great deal of space, and the pictures are easy to be copied. It may result in letting out secret. If the pictures are transferred into binary data flow to store in the database, this problem can be avoided.

2) Realization method: The collection of binary data is regarded as BLOB(Binary Large Object Block) from the aspect of storage. In SQL Server, the length of Image data is an uncertain binary data. The maximal length of it is $2 \mathrm{~GB}$. It is enough to store ordinary picture data, which can store pictures information of various formats. By using binary transformation methods, the system stored all pictures information data into image fields, and it will be transferred into its original format by certain procedure when the picture is needed to use, from which the overall data storage was realized and the data failure from platform replacement and data transplantation was avoided. The procedure of storing image is shown in Fig. 6. 


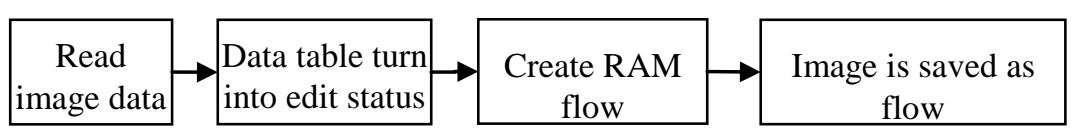

Fig.6 The procedure of storing image

\section{Conclusions}

The problem of irregular elements filling, fragmented data and unmatched material catalogue information between upper and subordinate unit in existed equipment maintenance material compilation catalogue was solved by developing and designing the equipment maintenance material catalogue compilation management system. The query census function of the system has improved the low efficiency and accuracy in analyzing big volume data created by manual analysis. The information management for compiling equipment maintenance material catalogue was realized. It is of great significance for equipment maintenance material catalogue to promote compilation efficiency to realize the standardization and intellectualization of material catalogue compilation. It has both military and economy efficiency.

\section{Reference}

[1] Li Xia, Liang Zhi-hao, Wang Wei. Some Thoughts on Constructing the Standard System of Equipment Maintenance Material Management[J]. Power Supply Technology and Applications, $2013(9): 35$.

[2] Dai Dongsheng, Ji Yalin, Lin Huijie. Flow design for equipment maintenance material management [J]. Journal of Sichuan Ordnance,2009(11):65.

[3] Zhang Sheng, Liu Jianguo You Wei. Dimensional Modeling of Maintain Equipment Data Warehouse [J]. Logistics Sci-Tech. 2008, (10): 38.

[4] Zhang Hongju. Research on information management technology for weapon maintenance material [D] . Changsha: National University of Defense Technology, 2003.

[5] Cai Xiaoyan, Li Longteng, GeYu. Research on realization of import and export data of Excel based on C\# [J]. Intelligent Computer and Application, 2014,4(5):83.

[6] Li Mingming. Research of Data Import to Excel in Background Thread Based on VS[J]. Net Platform. Measure and Control Technology, 2011, 30 (8) : 83.

[7] Su Qing, Li Zhongliang, Wu Weiming. Research on Method of Export Data into Excel in C\#.NET [J]. Computer and Modernization,2011(4):29.

[8] Wang Sen. Research and Implementation of Excel Importing Technology Based on C\# [J]. Office Automation, 2011 (16) : 54.

[9] Fan Yu. Use of COM Components Let the Data in the SQL Server Output to Excel [J]. Computer Programming Skills and Maintenance,2009(20:) 65.

[10] Wang Sheng. Analysis on Classic Example of Database Development [M] . Beijing: Tsinghua University Press, 2010:65. 